Research Paper

\title{
Suppression of non-small cell lung cancer migration and invasion by hsa-miR-486-5p via the TGF- $/$ /SMAD2 signaling pathway
}

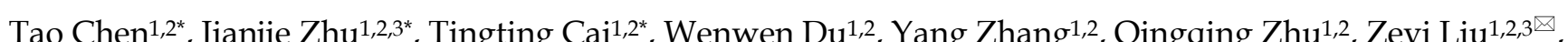
Jian-an Huang $1,2,3 \bowtie$

1. Department of Respiratory Medicine, the First Affiliated Hospital of Soochow University, Suzhou 215006, China

2. Suzhou Key Laboratory for Respiratory Diseases, Suzhou 215006, China

3. Institute of Respiratory Diseases, Soochow University, Suzhou 215006, China

*These authors contributed equally to this work

$\bowtie$ Corresponding author: Zeyi Liu, Email: liuzeyisuda@163.com; Jian-an Huang, Email: huang_jian_an@163.com

(C) The author(s). This is an open access article distributed under the terms of the Creative Commons Attribution License (https://creativecommons.org/licenses/by/4.0/). See http://ivyspring.com/terms for full terms and conditions.

Received: 2019.03.19; Accepted: 2019.08.18; Published: 2019.10.15

\begin{abstract}
Non-small cell lung cancer (NSCLC) is the leading cause of cancer-related death worldwide. SMAD family member 2 (SMAD2) is a key element downstream of the transforming growth factor beta (TGF- $\beta$ ) signaling pathway that regulates cancer metastasis by promoting the epithelial-mesenchyme transition (EMT). MicroRNA miR-486-5p is a tumor suppressor in NSCLC progression. However, it remains unclear whether miR-486-5p is implicated in TGF- $\beta$ signaling and EMT in NSCLC. In the present study, high expression of SMAD2 mRNA was detected in NSCLC tissues and cell lines, and was associated with poor survival of patients with NSCLC. By contrast, miR-486-5p was downregulated in NSCLC tissues and cell lines. In silico prediction showed that SMAD2 was a potential target of miR-486-5p. The prediction was verified using a dual-luciferase reporter assay. Transwell assays showed that knockdown of SMAD2 inhibited TGF- $\beta$-induced EMT and migration and invasion in NSCLC cells. Similarly, miR-486-5p overexpression suppressed TGF- $\beta$-induced EMT and migration and invasion of NSCLC cells. The present study provides a new insight into the role of miR-486-5p in regulating TGF- $\beta$-mediated EMT and invasion in NSCLC.
\end{abstract}

\section{Introduction}

Non-small cell lung cancer (NSCLC) is the leading cause of cancer-related death worldwide [1, 2]. Although a variety of studies have been conducted on NSCLC cell proliferation, migration, and invasion, the mechanism of NSCLC progression remains unclear and the five-year survival rate of patients with NSCLC remains below 15\% [3]. More than $90 \%$ of deaths from solid tumors, including NSCLC, are mainly attributed to metastasis [4]. Hence, it is important to have a good understanding of the mechanisms underlying NSCLC metastasis.

Cell motility is important for tumor cell metastatic dissemination from the primary location to lymph or blood vessels. Transforming growth factor- $\beta$ (TGF- $\beta$ ) is a cytokine with multiple functions, such as in cell proliferation, differentiation, apoptosis, and cell motility [5, 6]. In particular, TGF- $\beta$-mediated signaling is markedly altered in NSCLC, and is functionally associated with the tumorigenic and metastatic processes of this disease $[7,8]$. Notably, accumulating evidence suggests that TGF- $\beta$ signaling is a potent inducer of the epithelial-mesenchymal transition (EMT) in various cancers, including NSCLC [9-11]. EMT is vital for morphogenesis during embryonic development and for the conversion of early-stage tumors into invasive malignancies [12,13], which is marked by the repression of E-cadherin expression and the induction of N-cadherin, Vimentin, and Snail expression [11,14]. SMAD family member 2 (SMAD2) has been identified as a key 
element downstream of the TGF- $\beta$ signaling pathway in regulating cancer metastasis through promoting EMT [15].

MicroRNAs (miRNAs) are a class of small noncoding RNAs that play essential roles in tumor development and progression via the regulation of various signaling networks associated with multiple cellular functions, such as cell proliferation and invasion [16-19]. Growing evidence from comprehensive expression analyses shows that many miRNAs are closely related to the development of human lung cancer [20, 21]. In our previous analysis of miRNA arrays we found that the expression of miR-486-5p was significantly downregulated in NSCLC tissues [22].

In the present study, to identify new targets of miR-486-5p that may play a role in NSCLC, we predicted its target mRNAs using computational algorithms. Interestingly, the 3'-UTR of SMAD2 mRNA is a direct target of miR-486-5p. Furthermore, we found that ectopic application of miR-486-5p could inhibit TGF- $\beta$-induced EMT and invasion of NSCLC cells. We also discovered a novel mechanism and target of miR-486-5p in NSCLC. MiR-486-5p that directly suppressed SMAD2 expression, and overexpression of miR-486-5p inhibited the migratory ability of NSCLC cells. Our study provides a new insight into miR-486-5p's regulation of metastasis in NSCLC.

\section{Materials and Methods}

\section{Tissue samples}

Paired NSCLC tissue and adjacent noncancerous lung tissue samples (65 of each) were collected with the informed consent of the patients from the First Affiliated Hospital of Soochow University between 2012 and 2015. The patients had been diagnosed with NSCLC based on their histological and pathological characteristics according to the Revised International System for Staging Lung Cancer. They had not undergone chemotherapy or radiotherapy prior to tissue sampling. The tissue samples were snap frozen and stored in a cryofreezer at $-80^{\circ} \mathrm{C}$. This study was approved by the Ethics Committee of the First Affiliated Hospital of Soochow University.

\section{Cell Lines and Culture and Transfection}

Six human NSCLC cell lines A549, H1299, SPC-A1, H1650, H460 and H226, and one normal human bronchial epithelial cell BEAS-2B were obtained from the Cell Bank of the Chinese Academy of Sciences (Shanghai, China). The cells were grown in Roswell Park Memorial Institute (RPMI) 1640 (HyClone, South Logan, UT)medium containing 10\% fetal bovine serum (FBS) (Gibco, Carlsbad, CA, USA),
L-glutamine and penicillin-streptomycin (Invitrogen, Carlsbad, CA, USA) at $37{ }^{\circ} \mathrm{C}$ in a humidified atmosphere containing $5 \% \mathrm{CO}_{2}$.

miR-486-5p mimics, miR-486-5p inhibitor and negative control mimics, inhibitor were obtained from GenePharma (Shanghai, China). The SMAD2 siRNAs and negative control siRNA were designed by Ribobio (Guangzhou, China), the sequences were as flows siRNA-1: GGUGAAGAAGCUAAAGAAATT, siRNA-2: CAGGCCUUUACAGCUUCUCTT. The mimics, inhibitor or siRNA were transfected into A549 and H226 cells by Lipofectamine ${ }^{\mathrm{TM}} 2000$ reagent (Invitrogen, CA) according to the manufacturers' protocols.

\section{RNA Extraction, cDNA Synthesis, and Quantitative Real-time PCR (qRT-PCR)}

Total RNA wasextracted from cells and tissues using RNAiso Plus reagent was extracted (Takara, Osaka, Japan) according to the manufacturer's protocol. The RNA concentration was measured using a NanoDrop 2000 instrument (Thermo Fisher Scientific, Waltham, MA, USA). Synthesis of cDNA was performed using Reverse Transcriptase M-MLV (Takara). The primers for reverse transcription and amplification of miR486-5p and U6 were designed and synthesized by Guangzhou RiboBioCorp (Guangzhou, China). The sequences of the primers for qRT-PCR of SMAD2 and ACTB ( $\beta$-actin) were as follows: SMAD2 Forward: 5'-CAGCCATC-GTTGTCCACT-3', Reverse: 5'-GCTG GGGTGCTGTATGTC-3', $\beta$-actin mRNA, Forward: 5'-CACAGAGCCTCGCCTTTGCC-3', Reverse: 5'-AC CCATGCCCACCATCACG-3'. the primers for U6 was purchased from RiboBioCo. Ltd. (Guangzhou, China). qRT-PCR was performed using SYBR Premix ExTaq ${ }^{\mathrm{TM}}$ (Takara)according to the manufacturer's instructions on an ABI Step One Plus Real-Time PCR system (Applied Biosystems). The expression values of SMAD2 mRNA and miR-486-5p were normalized to internal controls $A T C B$ and U6, respectively. Relative expression was calculated using the ${ }^{\Delta \Delta} \mathrm{Ct}$ method [23].

\section{Western Blotting Assay}

Cells were grown to 80-90\% confluence and then lysed in RIPA buffer (Cell Signaling Technology, Danvers, MA, USA) with protease inhibitors and a phosphatase inhibitor cocktail (Sigma-Aldrich, St. Louis, MO, USA). The samples were centrifuged at $12,000 \mathrm{~g}$ for $15 \mathrm{~min}$, and the total cell lysates were separated by $10 \%$ SDS-PAGE electrophoresis and transferred to nitrocellulose membranes (Millipore, Billerica, MA, USA). The membranes were blocked with $5 \%$ bovine serum albumin (BSA) in Tris-buffered saline- $0.1 \%$ Tween-20 (TBST) buffer for $1 \mathrm{~h}$ at room 
temperature, incubated with primary antibodies overnight at $4{ }^{\circ} \mathrm{C}$, and then incubated with the corresponding horseradish peroxidase (HRP)conjugated secondary antibodies for $2 \mathrm{~h}$ at room temperature. Detection was performed using an ECL kit (Pierce, Rockford, IL, USA). The band density was quantified using Quantity One 4.6 software. The antibodies used for western blotting, including anti-Smad2, anti-pSmad2, Snail, MMP2, anti- $\beta$-actin, anti-mouse and anti-goat secondary antibodies were purchased from Cell Signaling Technology. Antibodies targeting E-Cadherin, N-Cadherin, Vimentin were obtained from BD Biosciences.

\section{Plasmid Construction, Transient Transfection, and Luciferase Assay}

A 225-bp fragment of the SMAD2 3'-UTR containing the miR-486-5p target sites (positions 304-310), as predicted by TargetScan (http://www. targetscan.org/vert_72/) or mutated sites, was synthesized and fused to the 3'-end of a psiCHECK2 dual-luciferase reporter vector (Promega, Madison, WI, USA). A549 and H226 cells were plated in a $24-w e l l$ plate. The constructed reporter plasmids were co-transfected with either miR-486-5p mimics or a negative control (miR-NC) into the cells using Lipofectamine 2000 (Life Technologies, Carlsbad, CA, USA). The plates were maintained for $48 \mathrm{~h}$, and then the cells were collected and the luciferase activity was measured using a Dual-Luciferase Reporter Assay kit (Promega). Each experiment was conducted in triplicate.

\section{Wound Healing Assay}

A wound healing assay was performed as described previously [24]. Briefly, A549 and H226 cells were seeded into 6-well tissue culture plates after $24 \mathrm{~h}$ of transfection and cultured until they reached $\sim 80-90 \%$ confluence as a monolayer. The monolayer was gently and slowly scratched using a fresh $10-\mu 1$ pipette tip across the center of the well. The resulting gap distance should equal the outer diameter of the end of the tip. Another scratch was made perpendicular to the first to create a cross in each well. The cells were then washed gently twice with $1 \times$ PBS to remove the detached cells. The well was replenished with fresh medium and the cells were grown for an additional $24 \mathrm{~h}$. The cells were observed and photographed under a microscope(CKX41, Olympus) at the same distance and settings. The width of the gap was evaluated quantitatively using Photoshop.

\section{Migration and Invasion Assays}

Transwell migration and invasion assays were performed as described previously [24]. Briefly, $5 \times$
$10^{4}$ A549 and H226 cells transfected with miRNAs or short interfering RNAs (siRNA) were added to the upper chamber of Transwell plates (BD Biosciences, San Jose, CA, USA) in the presence of $1 \%$ FBS in medium. At the same time, 10\% FBS medium was added as a chemoattractant to each lower chamber. If necessary, TGF- $\beta 1$ ( $5 \mathrm{ng} / \mathrm{mL})$ was added to the upper chamber $6 \mathrm{~h}$ later. Cells were allowed to migrate through an $8-\mu \mathrm{m}$ pore membrane or invade a membrane coated with Matrigel. After $24 \mathrm{~h}$ of incubation, the cells that had migrated onto the lower surface of the chamber were fixed with 100\% methanol and stained with $1 \%$ crystal violet. Finally, the cells were counted in least three random fields under a light microscope.

\section{Statistical Analysis}

An unpaired $t$ test (two-tailed) was used to analyze the significance of the data from the cells. A paired $t$ test (two-tailed) was performed to determine the significance of the data from patient samples. $P<$ 0.05 was considered a significant difference. Statistical analyses were conducted using GraphPad Prism 7 software (GraphPad, San Diego, CA, USA).

\section{Results}

\section{SMAD2 is upregulated in NSCLC tissues and cell lines}

Public data from Gene Expression Omnibus (GSE19188) showed that SMAD2 mRNA expression was significantly upregulated in lung carcinoma compared with that in normal lung tissues (Figure $1 \mathrm{~A})$. We verified the expression of SMAD2 mRNA in 65 paired NSCLC tissues and adjacent noncancerous lung tissues and found that SMAD2 mRNA levels were significantly higher in NSCLC tissues than in the adjacent noncancerous lung tissues $(P<0.05$, Figure $1 \mathrm{~B}$ and Table 1). Patient characteristics with respect to the decreased expression of SMAD2 and miR-486-5p are shown in Table 1. No significant difference in the SMAD2 and miR-486-5p mRNA level was observed in the NSCLC samples age, gender, histology, lymph node status, smoking history and distant metastases. In addition, the public dataset from Kaplan-Meier Plotter (http:/ / www.kmplot.com) indicated that high expression of SMAD2 was significantly associated with poor survival of patients with NSCLC $(P<0.01$, Figure 1C). Next, we detected SMAD2 mRNA and protein levels in six NSCLC cell lines and in a bronchial epithelial cell line, BEAS-2B, using qRT-PCR and western blotting analysis (Figure 1D). The results showed that the SMAD2 levels in the NSCLC cell lines were higher than those in the BEAS-2B cells. Collectively, our data showed that SMAD2 is 
upregulated in NSCLC tissues and cell lines.
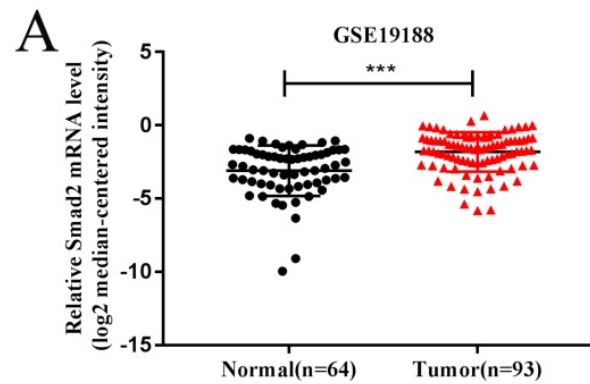

$\mathrm{C}$

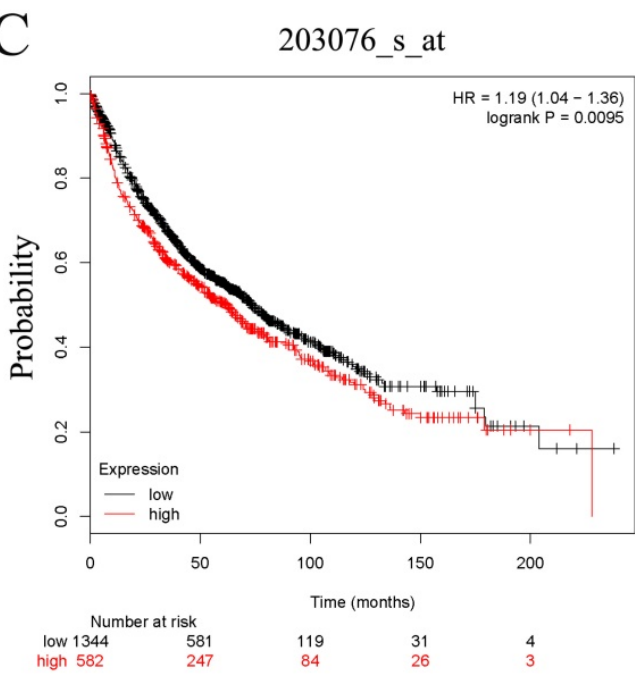

B

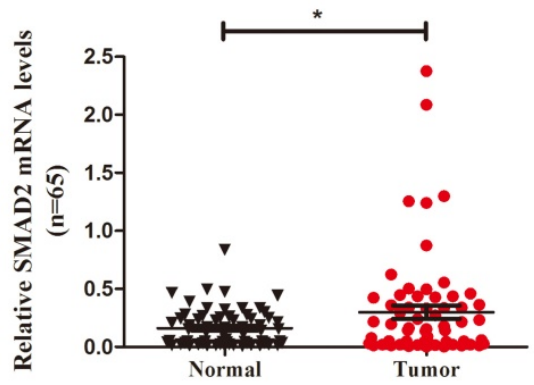

$\mathrm{D}$

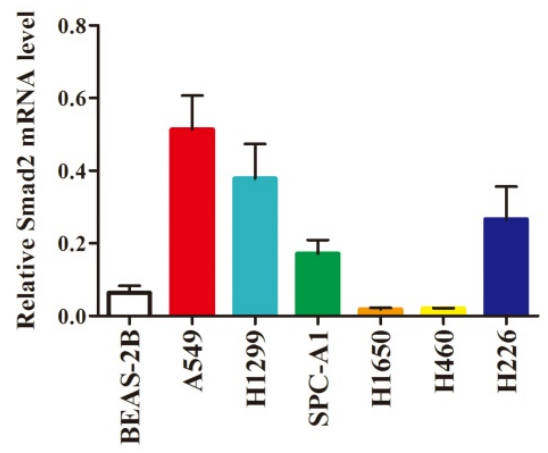

Smad2

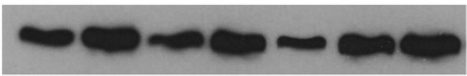

$\beta$-actin

Figure 1. SMAD2 is upregulated in NSCLC tissues and cell lines. (A) Data obtained from GEO (GSE19188) were analyzed to compare the expression difference of SMAD2 between NSCLC tissues and noncancerous lung tissues. (B) The mRNA expression levels of SMAD2 were determined by qRT-PCR, and the data were compared between 65 NSCLC and paired adjacent noncancerous lung tissues.(C) The relationship between the expression levels of SMAD2 and overall survival for 1926 NSCLC patients were analyzed, and Kaplan-Meier plots were generated using the Kaplan-Meier Plotter (http://www.kmplot.com).(D) Total RNA and protein were extracted from several cell lines, and the levels of SMAD2 mRNA and protein were detected by QRT-PCR and western blotting assay, respectively. $\beta$-actin was used as an internal control. Data are shown as the mean $\pm \mathrm{SE} . *, * *$, and $* * *$ indicate significant differences compared with the control $(* P<0.05 ; * * P<0.01 ; * * * P<0.001)$.

Table 1. Demographic and clinical characteristics of NSCLC patients and the level of miR-486-5p and Smad2 mRNA expression in tumor tissue specimens

\begin{tabular}{|c|c|c|c|c|c|}
\hline Characteristics & Number of cases (\%) & miR-486-5p & $P$ value & Smad2 mRNA & $P$ value \\
\hline \multicolumn{6}{|l|}{ Age (years) } \\
\hline$\leq 65$ & $30(46.2 \%)$ & $0.0034 \pm 0.0010$ & 0.756 & $0.1888 \pm 0.0505$ & 0.070 \\
\hline$>65$ & $35(53.8 \%)$ & $0.0042 \pm 0.0023$ & & $0.3947 \pm 0.0938$ & \\
\hline \multicolumn{6}{|l|}{ Gender } \\
\hline Male & $42(64.6 \%)$ & $0.0046 \pm 0.0021$ & 0.467 & $0.3690 \pm 0.0838$ & 0.098 \\
\hline Female & $23(35.4 \%)$ & $0.0025 \pm 0.0006$ & & $0.1730 \pm 0.0374$ & \\
\hline \multicolumn{6}{|l|}{ Histological features } \\
\hline Adenocarcinoma & $34(52.3 \%)$ & $0.0032 \pm 0.0009$ & 0.142 & $0.2905 \pm 0.0607$ & 0.967 \\
\hline Squamous cell carcinoma & $25(38.5 \%)$ & $0.0054 \pm 0.0033$ & & $0.3178 \pm 0.1227$ & \\
\hline Others & $6(9.2 \%)$ & $0.0007 \pm 0.0003$ & & $0.2762 \pm 0.0789$ & \\
\hline \multicolumn{6}{|l|}{ Degree of differentiation } \\
\hline Low & $25(38.5 \%)$ & $0.0017 \pm 0.0004$ & 0.211 & $0.2271 \pm 0.0619$ & 0.315 \\
\hline Middle & $40(61.5 \%)$ & $0.0052 \pm 0.0022$ & & $0.3451 \pm 0.0835$ & \\
\hline \multicolumn{6}{|l|}{ Smoker } \\
\hline Yes & $34(52.3 \%)$ & $0.0053 \pm 0.0025$ & 0.274 & $0.3461 \pm 0.0948$ & 0.395 \\
\hline No & $31(47.6 \%)$ & $0.0023 \pm 0.0005$ & & $0.2487 \pm 0.0581$ & \\
\hline \multicolumn{6}{|l|}{ Clinical stage } \\
\hline $\mathrm{I}+\mathrm{II}$ & $33(50.8 \%)$ & $0.0051 \pm 0.0025$ & 0.359 & $0.2653 \pm 0.0730$ & 0.543 \\
\hline III + IV & $32(49.2 \%)$ & $0.0026 \pm 0.0008$ & & $0.3351 \pm 0.0880$ & \\
\hline \multicolumn{6}{|l|}{ Distant metastasis } \\
\hline No & $56(86.2 \%)$ & $0.0040 \pm 0.0015$ & 0.744 & $0.2571 \pm 0.0576$ & 0.060 \\
\hline Yes & $9(13.8 \%)$ & $0.0027 \pm 0.0017$ & & $0.5649 \pm 0.1833$ & \\
\hline \multicolumn{6}{|l|}{ Lymph node metastasis } \\
\hline No & $35(53.8 \%)$ & $0.0052 \pm 0.0024$ & 0.283 & $0.3509 \pm 0.0841$ & 0.334 \\
\hline Yes & $30(46.2 \%)$ & $0.0022 \pm 0.0008$ & & $0.2399 \pm 0.0740$ & \\
\hline
\end{tabular}

Data are presented as mean \pm SEM. Kruskal-Wallis test for comparison between three or more groups. 

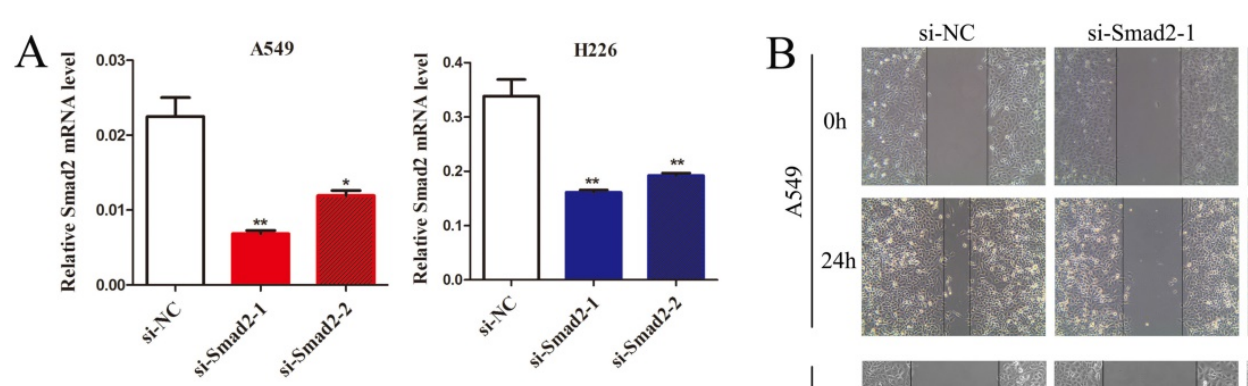

si-Smad2-2

$\mathrm{C}$

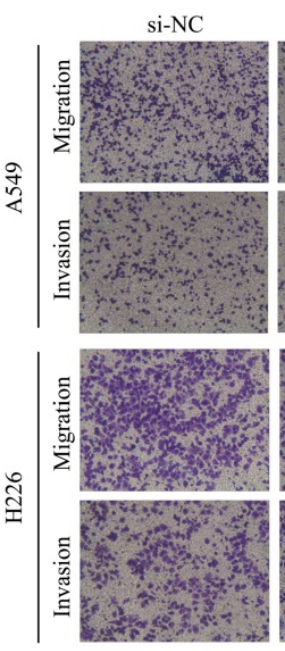

si-Smad2-1
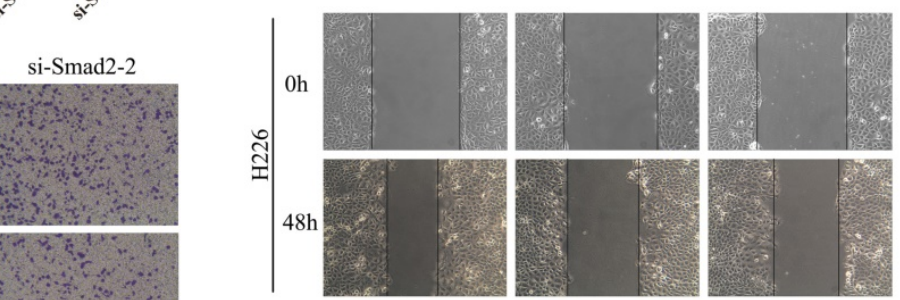

D

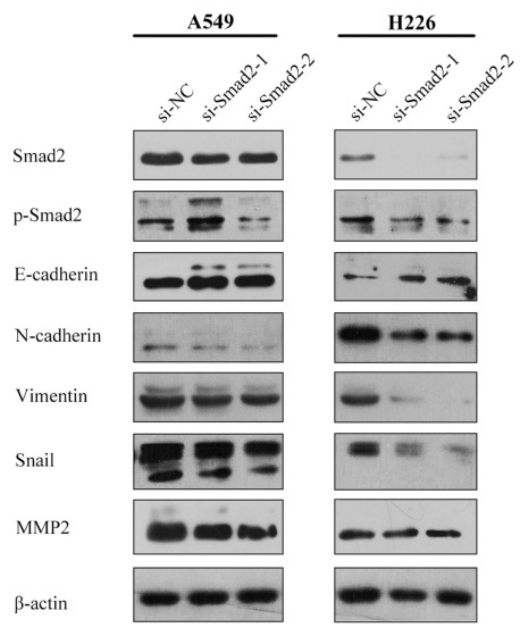

Figure 2. Knockdown of SMAD2 inhibits cell EMT and the migration and invasion of NSCLC cells. (A) Si-RNAs against SMAD2 were transfected into A549 (left) and $\mathrm{H} 226$ (right) cells for $48 \mathrm{~h}$. The cells were subjected to determination of SMAD2 mRNA expression using qRT-PCR. (B) A uniform scratch was made in each confluent monolayer of $\mathrm{A} 549$ (upper) cells and $\mathrm{H} 226$ (bottom) cells transfected with siRNAs for SMAD2. Images were acquired at $0 \mathrm{~h}$ and $24 \mathrm{~h}$ post scratching under a microscope. (C) A549 and $\mathrm{H} 226$ cells knocked down for SMAD2 were allowed to migrate through an 8- $\mu$ m pore in a Transwell apparatus. Migrated cells were stained and counted in at least three microscopic fields $24 \mathrm{~h}$ later. One representative image (upper) is shown, and the number of migrated cells was compared between groups of SMAD2-silenced and negative control (bottom). After transfection with SMAD2 siRNAs for $48 \mathrm{~h}$, A549 and $\mathrm{H} 226$ cells were subjected to western blotting to determine the expression of various proteins. $\beta$ -actin was used as an internal control. Data are shown as the mean \pm SD. $*$, **, and $* * *$ indicate significant differences compared with the control $(* P<0.05 ; * * P<0.01 ; * * * P<$ $0.001)$.

\section{Knockdown of SMAD2 inhibits cell EMT and the migration and invasion of NSCLC cells}

SMAD2 is an important element of the TGF- $\beta$ signaling pathway that plays a critical role in cell EMT and invasion. Hence, we first observed the influence of knockdown of SMAD2 on the cell migration and invasion of NSCLC cells. Two siRNAs were used to inhibit SMAD2 expression in A549 and H226 cells (Figure 2A). Wound healing tests showed that knockdown of SMAD2 markedly inhibited the migratory capacity of A549 and H226 cells (Figure 2B). As shown in Figure 2C, in Transwell assays, cell migration and invasion were significant suppressed in both A549 and H226 cells after knockdown of SMAD2. Moreover, EMT was inhibited by knockdown of SMAD2 in the NSCLC cells, which was manifested as downregulated levels of p-SMAD2, N-cadherin, Vimentin, Snail, and matrix metallo- proteinase 2 (MMP2), and upregulated E-cadherin, compared with their levels in the negative control cells (Figure 2D). Taken together, the results showed that SMAD2 is involved in EMT and invasion of NSCLC cells.

\section{Knockdown of SMAD2 represses TGF- $\beta$-induced cell EMT, and migration and invasion in NSCLC cells}

TGF- $\beta 1$ was introduced and cell migration and invasion were determined. TGF- $\beta$ induced the migration and invasion of A549 cells, which was inhibited by knockdown of SMAD2 (Figure 3A, upper panel). The results were similar in $\mathrm{H} 226$ cells (Figure $3 \mathrm{~A}$, lower panel). TGF- $\beta$-induced EMT was estimated by detecting several EMT markers that could be regulated by TGF- $\beta$. As shown in Figure 3B and C, treatment with TGF- $\beta 1$ caused levels of E-cadherin to 
decrease, while $\mathrm{N}$-cadherin, Vimentin, Snail, and MMP2 levels increased in A549 (Figure 3B) and H226 cells (Figure 3C). However, the TGF- $\beta 1$-induced variation in EMT maker levels was inhibited by
siRNA-mediated knockdown of SMAD2 in A549 (Figure 3B) and H226 cells (Figure 3C). These results showed that knockdown of SMAD2 expression repressed TGF- $\beta 1$-induced EMT in NSCLC cells.
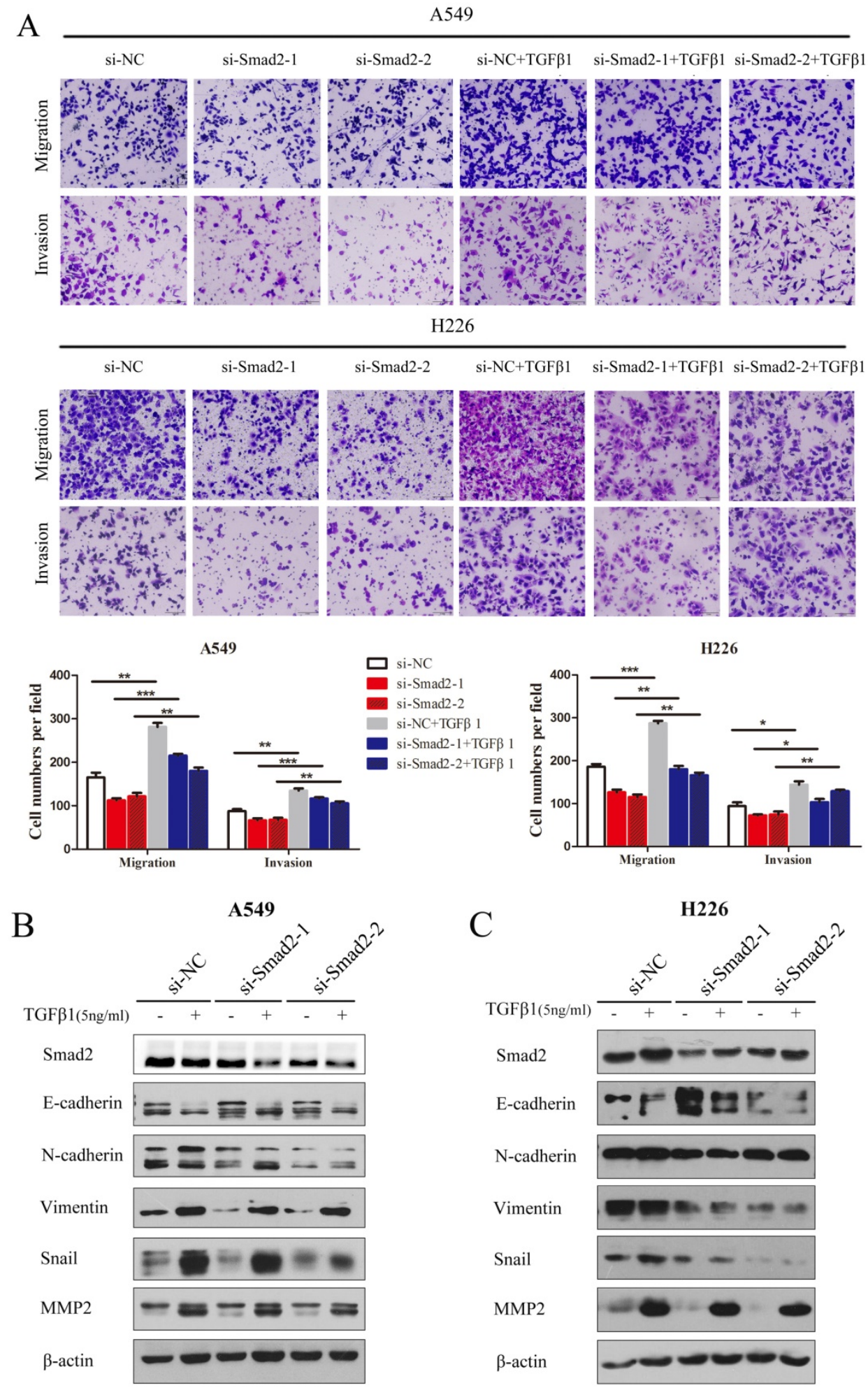

Figure 3. Knockdown of SMAD2 represses TGF- $\beta$-induced EMT, and migration and invasion in NSCLC cells. (A) In the presence or absence of TGF- $\beta 1$ ( 5 $\mathrm{ng} / \mathrm{mL}$ ), A549 and H226 cells knocked down for SMAD2 were allowed to migrate through an 8- $\mu \mathrm{m}$ pore in a Transwell apparatus. One day later, migrated cells were stained and counted in at least three microscopic fields. One representative image (upper) is shown, and the number of migrated cells was compared between groups of si-SMAD2 and si-NC in the presence of TGF- $\beta 1$ (bottom).(B and C) A549 (B) and H226 (C) cells transfected with siRNAs for SMAD2 or si-NC were treated with TGF- 31 (5 ng/mL) for 24 h, and then the expression of various proteins were determined by western blotting. $\beta$-actin was used as an internal control. Data are shown as the mean \pm SD. *, $* *$, and $* * *$ indicate significant differences compared with the control $(* P<0.05 ; * * P<0.01 ; * * * P<0.001)$ 
MiR-486-5p inhibits SMAD2 expression by $3^{\prime}$ UTR-binding, and is downregulated in NSCLC tissues and cell lines

In silico prediction (TargetScan) showed that $S M A D 2$ is a potential target of miR-486-5p. To verify this, a dual-luciferase reporter vector containing the SMAD2 3' UTR seed region specific to miR-486-5p, or the corresponding mutant sequence, was used. As shown in Figure 4A, the luciferase activities of the reporter vector containing the seed region of miR-486-5p in A549 and H226 cells was significantly

A

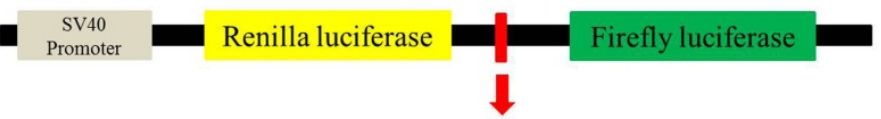

Smad2 3'-UTR WT

ACAGUAGUAGUUAUGUGUACAGGU

hsa-miR-486-5p

GAGCCCCGUCGA-GU-CAUGUCCU

| | |

Smad2 3'-UTR MUT

ACAGUAGUAGUUAUGUGUAGTCCU
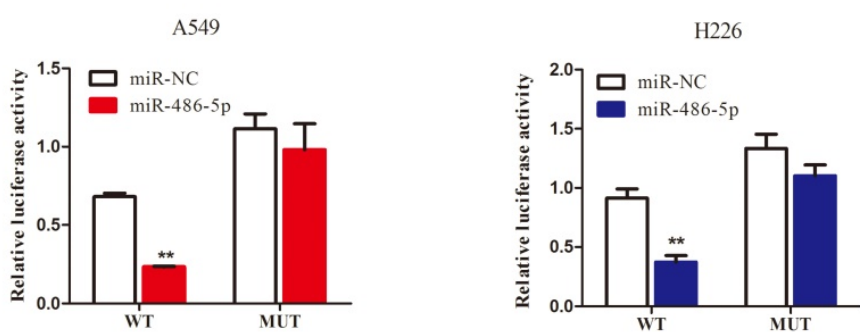

$\mathrm{B}$
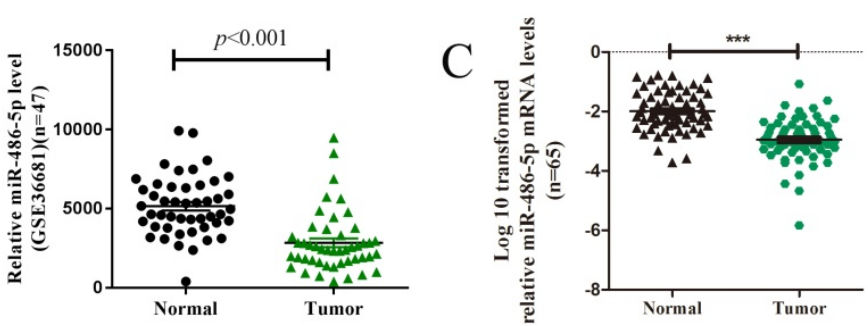

$\mathrm{D}$

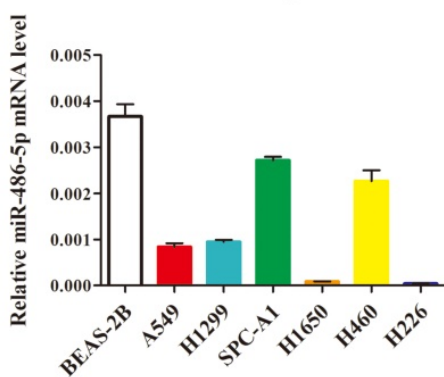

Figure 4. MiR-486-5p inhibits SMAD2 expression via binding to its $3^{\prime}$ UTR and is downregulated in NSCLC tissues and cell lines. (A) Schematic diagram showing the subcloning of the predicted miR-486-5p-binding site at position 304-310 of the SMAD2 3' UTR into a psiCHECK-2 luciferase construct. Predicted duplex formation between miR-486-5p and the wild-type or mutant miR-486-5p-binding site is indicated (upper). Luciferase activity of the construct containing the wild-type or mutant SMAD2 reporter gene in A549 (bottom, left) and $\mathrm{H} 226$ (bottom, right) cells co-transfected with the negative control (miR-NC) or miR-486-5p. Scrambled sequences were used as the NC. Relative Renilla luciferase activity was determined and normalized against firefly luciferase activity. (B) Data obtained from the GEO database (GSE36681) were analyzed to compare the expression of miR-486-5p between NSCLC tissues and noncancerous lung tissues. (C) The mRNA expression levels of miR-486-5p were determined using qRT-PCR, and the data were compared between $65 \mathrm{NSCLC}$ and paired adjacent noncancerous lung tissues. (D) qRT-PCR analysis of relative miR-486-5p expression in human NSCLC cell lines. Data are shown as the mean \pm SD. *,**, and *** indicate significant differences compared with the control $(* P<0.05$; ** $p<0.01$; *** $p<0.001)$. suppressed by miR-486-5p transfection compared with that in the cells transfected with miR-NC. However, the activities were restored when the seed region was mutated. Considering the increased expression of SMAD2 in NSCLC tissues and cell lines, we hypothesized that miR-486-5 $p$ might be decreased in NSCLC. As expected, the GEO data (GSE36681) showed that the expression of miR-486-5p was downregulated in NSCLC tissues. We also detected that the expression level of miR-486-5p was significantly decreased in 65 NSCLC tissues relative to their paired noncancerous tissues; these tissues were collected and stored by our research group. Consistent with the tissue expression data, miR-486-5p was also downregulated in several NSCLC cell lines compared with its level in BEAS-2B cells.

\section{MiR-486-5p represses SMAD2 expression, and the capability of migration and invasion of NSCLC cells}

A549 and H226 cells transiently transfected with miR-486-5p or the negative control (miR-NC) (Figure 5A, left panel) were subjected cell migration and invasion assays, and their expression of EMT markers was detected. SMAD2 mRNA expression was suppressed by miR-486-5p transfection in A549 and $\mathrm{H} 226$ cells (Figure 5A, right panel). Wound healing assays showed that cells transfected with miR-486-5p migrated slower than the control cells (Figure 5B). Similarly, Transwell assays showed that miR-486-5p inhibited the cell migration and invasion capability of A549 and $\mathrm{H} 226$ cells (Figure 5C). The different levels of several EMT markers related to cell migration were determined between the cells overexpressing miR-486-5p and miR-NC using western blotting. The results showed that overexpression of miR-486-5p in A549 and H226 cells significantly suppressed the protein levels of SMAD2 and phosphorylation of SMAD2 (p-SMAD2), and inhibited the EMT process, as reflected by the upregulation of E-cadherin and the downregulation of N-cadherin, Vimentin, Snail, and MMP2 levels. These results were in turn confirmed by transfection with miR-486-5p inhibitor mimics. As shown in Figure 6A, transfection of the miR-486-5p inhibitor mimics in A549 and H226 cells markedly inhibited the expression of miR-486-5p (left panel), and relieved the inhibition on SMAD2 mRNA expression induced by miR-486-5p (right panel). In contrast to miR-486-5p, the inhibitor mimics 
dramatically promoted cell migration and invasion, as determined by wound healing (Figure 6B) and Transwell assays (Figure 6C) in A549 and H226 cells. Meanwhile, inhibition of miR-486-5p significantly increased total SMAD2 and p-SMAD2 levels, and promoted the EMT process, as demonstrated by downregulation of E-cadherin and upregulation of N-cadherin, Vimentin, Snail, and MMP2 levels (Figure 6D). Taken together, these results showed that miR-486-5p inhibits SMAD2 activity, cell EMT, and cell migration in NSCLC.

\section{MiR-486-5p inhibits TGF- $\beta$-induced cell EMT, and migration and invasion in NSCLC cells}

TGF- $\beta$-induced cell EMT, we hypothesized that miR-486-5 $p$ would influence TGF- $\beta$-induced cell EMT and cell migration. As shown in Figure 7A, miR-486-5p significantly suppressed cell migration and invasion in both A549 and H226 cells in the presence of TGF- $\beta 1$ (Figure 7A). In addition H226 cells, the variation in the levels of EMTmarkers downregulation of E-cadherin, upregulation EMT markers of N-cadherin, Vimentin, Snail, and MMP2 were prevented by miR-486-5 $p$ overexpression in A549 and H226 cells (Figure 7B). These data are in line with the effect of knockdown of SMAD2 on TGF- $\beta$-induced EMT, suggesting an EMT-repressive role of miR-486-5p in NSCLC cells.

Considering that SMAD2 is implicated in
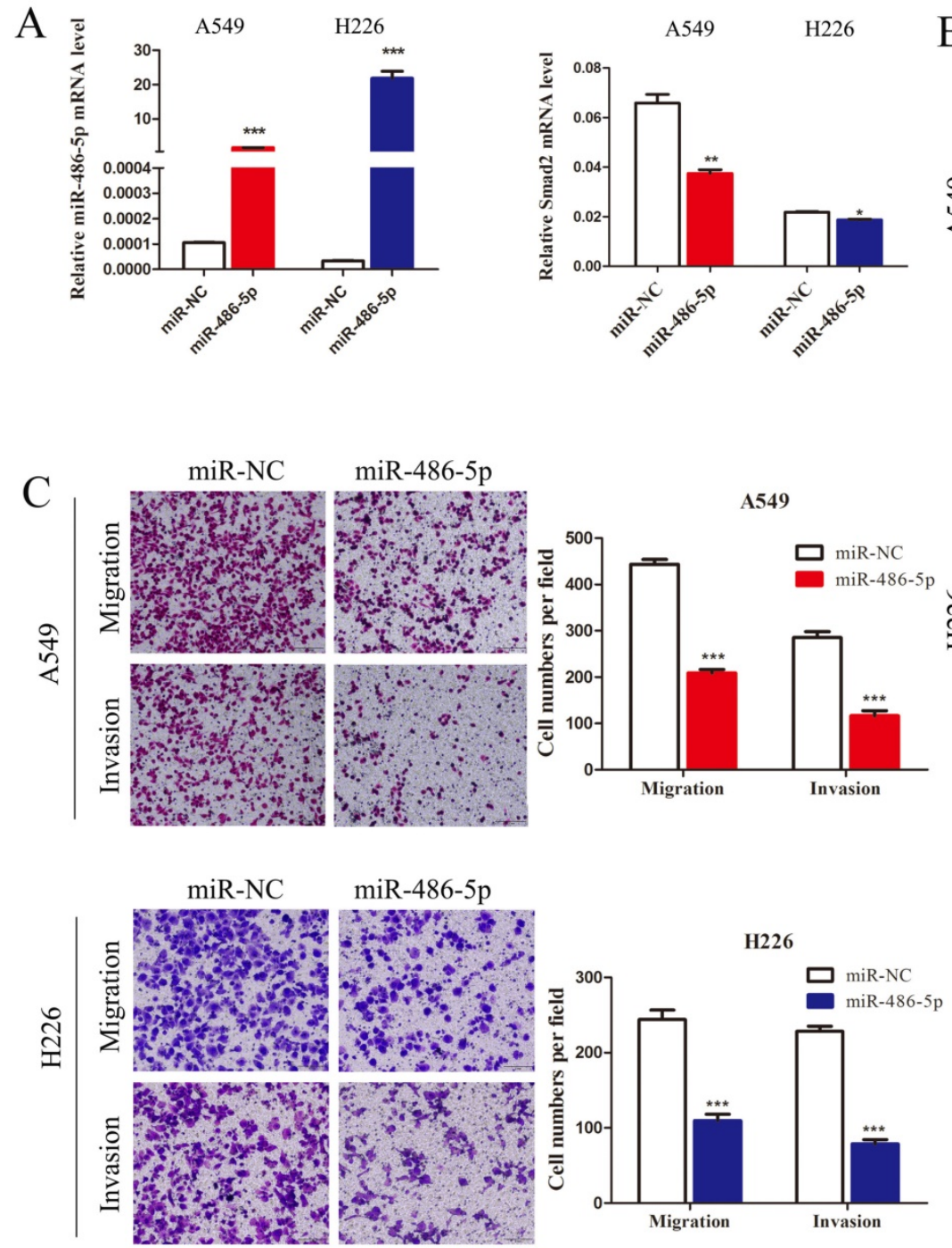
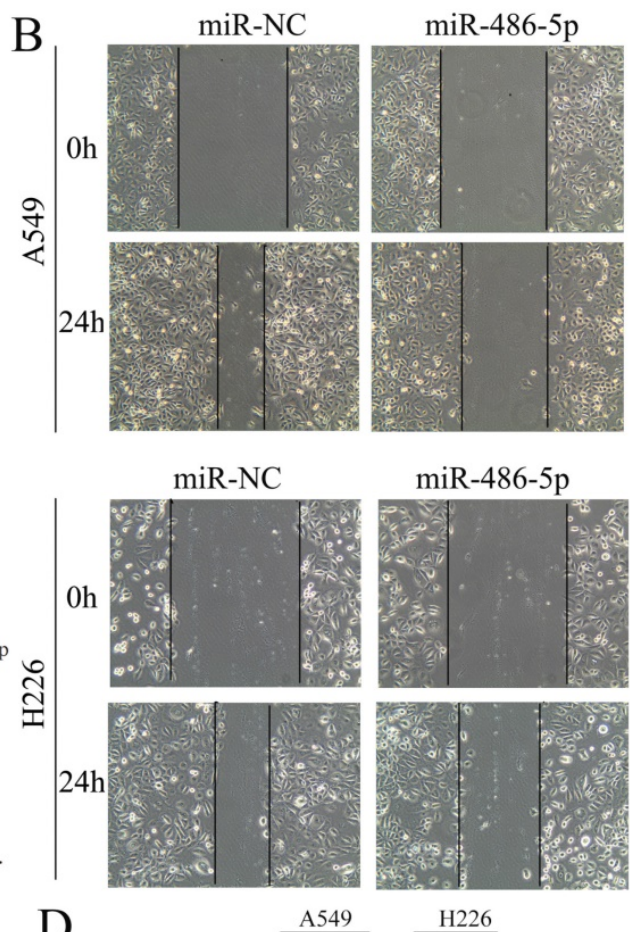

$\operatorname{miR}-486-5 \mathrm{p}$

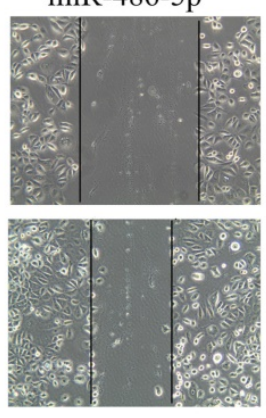

$\mathrm{D}$

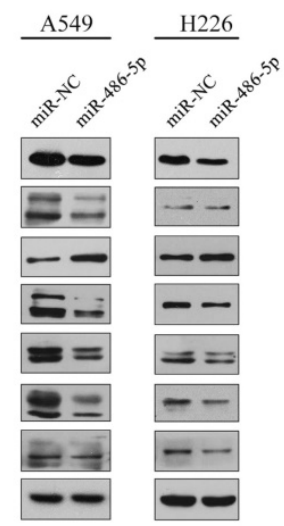

Figure 5. MiR-486-5p represses SMAD2 expression, and the migration and invasion abilities of NSCLC cells. (A) miR-486-5p mimics were transfected into A549 and $\mathrm{H} 226$ cells for $48 \mathrm{~h}$. The expression levels of miR-486-5p (left) and SMAD2 mRNA (right) were determined in the cells using qRT-PCR assay. (B) A uniform scratch was made in each confluent monolayer of A549 (upper) cells and $\mathrm{H} 226$ (bottom) cells transfected with miR-486-5p or miR-NC. Images were acquired at $0 \mathrm{~h}$ and $24 \mathrm{~h}$ post scratching under a microscope. (C) A549 and H226 cells transfected with miR-486-5p or miR-NC were allowed to migrate through an 8- $\mu \mathrm{m}$ pore in a Transwell apparatus. Migrated cells were stained and counted in at least three microscopic fields $24 \mathrm{~h}$ later. One representative image (left) is shown, and the number of migrated cells was compared between the miR-486-5p and miR-NC groups (right). After transfection with miR-486-5p for $48 \mathrm{~h}, \mathrm{~A} 549$ and $\mathrm{H} 226$ cells were subjected to western blotting to determine the expression of various proteins. $\beta$-actin was used as an internal control. Data are shown as the mean \pm SD. *, $* *$, and $* * *$ indicate significant differences compared with the control $(* P<0.05$; $* * P<0.01 ; * * * P<0.001)$. 


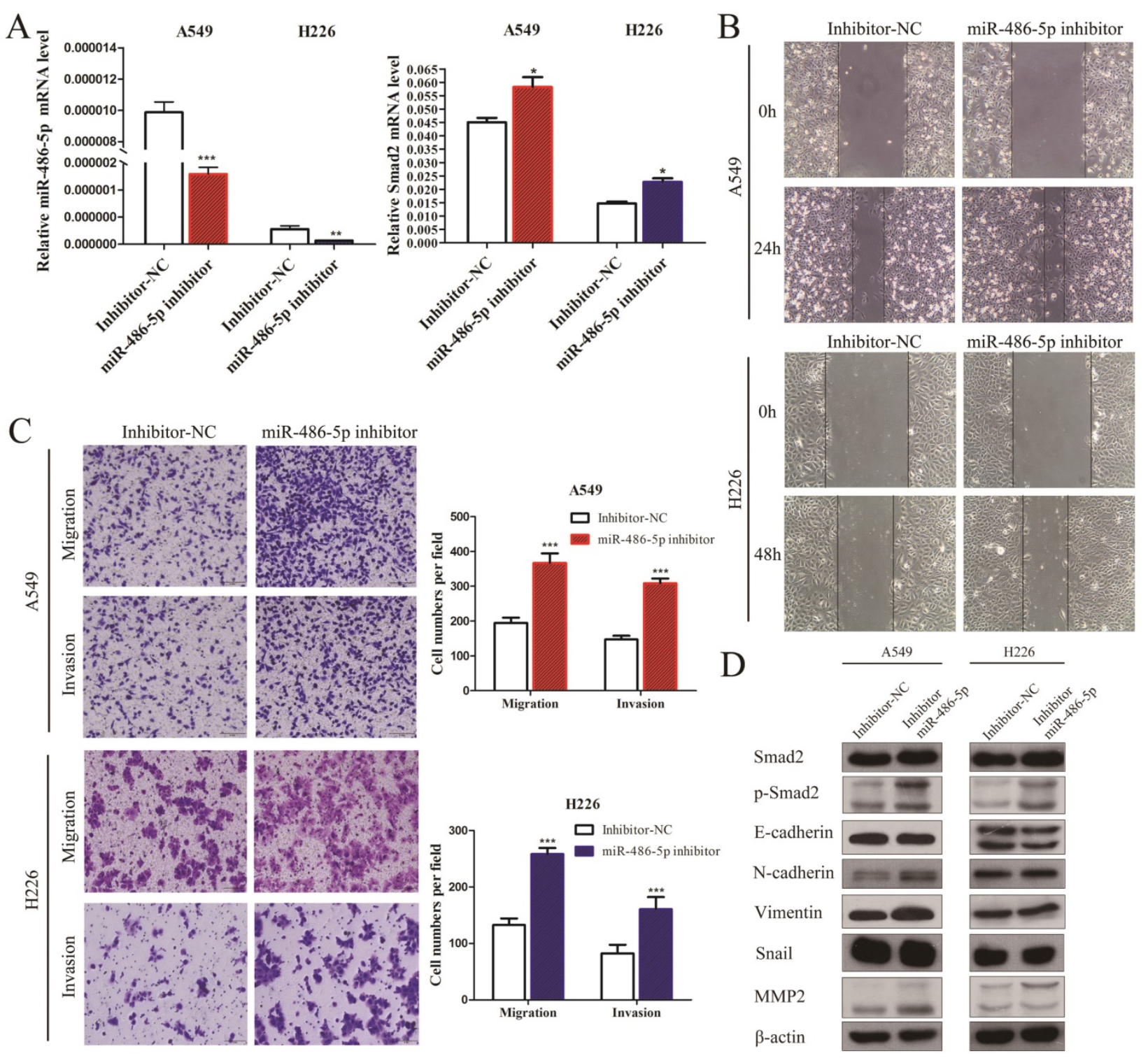

Figure 6. MiR-486-5p inhibitor enhances SMAD2 expression, and the capability of migration and invasion of NSCLC cells. (A) The miR-486-5p inhibitor mimics or negative control (inhibitor-NC) were transfected into A549 and H226 cells for $48 \mathrm{~h}$. The expression levels of miR-486-5p (left) and SMAD2 mRNA (right) were then determined using qRT-PCR. (B) A uniform scratch was made in confluent monolayers of A549 (upper) cells and H226 (bottom) cells the transfected of the miR-486-5p inhibitor or inhibitor-NC. Images were acquired at $0 \mathrm{~h}$ and $24 \mathrm{~h}$ post scratching under a microscope. (C) A549 and H226 cells transfected with the miR-486-5p inhibitor or inhibitor-NC were allowed to migrate through an 8-um pore in a Transwell apparatus. Migrated cells were stained and counted in at least three microscopic fields $24 \mathrm{~h}$ later. One representative image (left) is shown, and the number of migrated cells was compared between the miR-486-5p inhibitor and inhibitor-NC groups (right). After transfection with the miR-486-5p inhibitor for $48 \mathrm{~h}, \mathrm{~A} 549$ and $\mathrm{H} 226$ cells were subjected to western blotting to determine the expression of various proteins. $\beta$-actin was used as an internal control. Data are shown as the mean \pm SD. *, **, and $* * *$ indicate significant differences compared with the control $(* P<0.05 ; * * P<0.01 ; * * * P<0.001)$.

\section{Discussion}

Metastasis is the main reason for cancer related death from solid tumors, including NSCLC. The EMT process contributes markedly to the progress of tumor metastasis. The TGF- $\beta /$ SMADs signaling plays an essential role in accelerating EMT and cancer cell migration. As a member of the SMAD family, SMAD2 plays a key role, together with SMAD3 and SMAD4, in TGF- $\beta$ signaling and cancer progression [25]. In the present study, we found that SMAD2expression was upregulated in NSCLC tissues and cell lines, and that high levels of SMAD2mRNA were associated with poor prognosis of NSCLC, exhibiting an oncogenic potency in NSCLC. A previous study showed that silencing SMAD2 restrained TGF- $\beta$-induced EMT andcell invasion, indicating a therapeutic role for SMAD2 inhibition in cancer [26]. MiRNAs are small interfering RNA (siRNAs) with a length of about 20 bp that are produced in organisms. Not only can one miRNA can inhibit the expression of multiple genes, but also one gene can be targeted by multiple miRNAs. SMAD2 has been proven to be targeted and downregulated by various miRNAs such as miR-132 [27], miR-27a [28], and miR-323-3p [29] in regulating multiple cancers. 


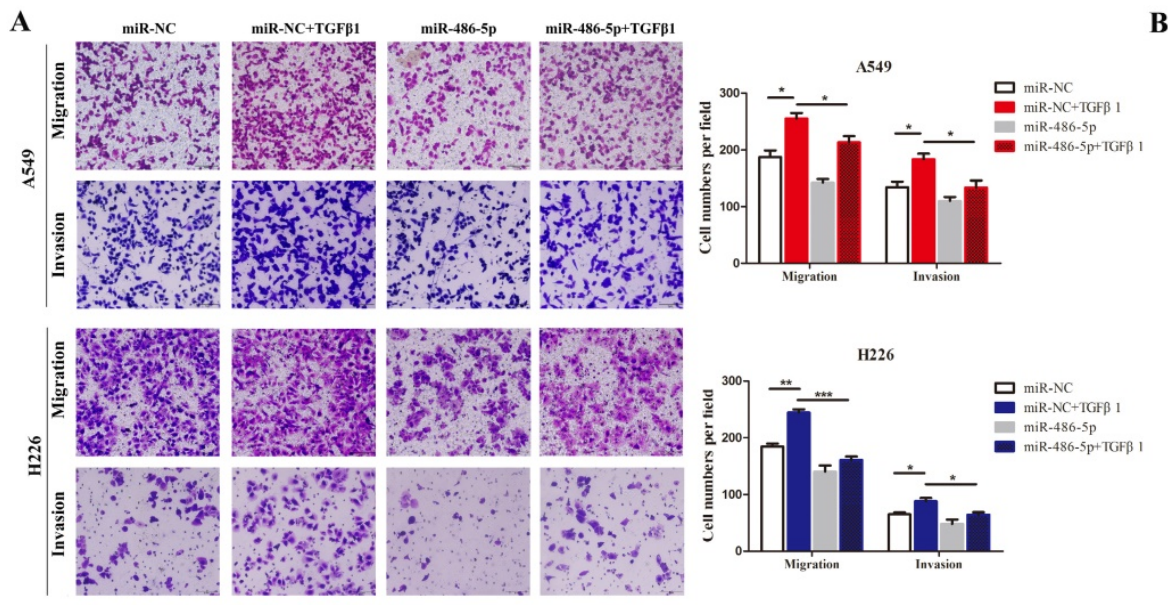

B

Figure 7. MiR-486-5p inhibits TGF- $\beta$-induced cell EMT, and migration and invasion in NSCLC cells. (A) In the presence or absence of TGF- $\beta 1$ ( $5 \mathrm{ng} / \mathrm{mL}), A 549$ and H226 cells transfected with miR-486-5p were allowed to migrate through an 8- $\mu \mathrm{m}$ pore in a Transwell apparatus. One day later, migrated cells were stained and counted in at least three microscopic fields. One representative image (upper) is shown, and the number of migrated cells was compared between the miR-486-5p and miR-NC groups in the presence of TGF- $\beta 1$ (bottom). (B and C) A549 (B) and H226 (C) cells transfected with miR-486-5p or miR-NC were treated with TGF- $\beta 1$ (5 ng/mL) for 24 h, and then the expression of various proteins was determined by western blotting. $\beta$-actin was used as an internal control. Data are shown as the mean \pm SD. *, **, and *** indicate significant differences compared with the control $(* P<0.05$; ** $P<0.01$; ***P $<0.001)$.

We observed an interaction between SMAD2 and miRNAs, such that SMAD2 expression was suppressed by the direct binding of miR-486-5p to the 3' UTR of SMAD2, providing a new insight into developing a therapeutic strategy for NSCLC. The interaction also suggested that miR-486-5p might have a great impact on cell EMT and migration in NSCLC. These results led to a similar conclusion to a recent study in lens epithelial cells that miR-486-5p prevents cell migration and invasion by targeting SMAD2 [30]. Moreover, a study in prostate cancer showed that miR-486-5p suppresses metastasis by targeting Snail and regulating the EMT process [31]. We found that miR-486-5p repressed TGF- $\beta$-induced EMT and cell migration and invasion in NSCLC cells.

Consistent with these proposed functions, we found that miR-486-5p expression was downregulated in NSCLC, which further confirmed a tumor suppressor role of miR-486-5p in cancer. Shao et al. recently reported that miR-486-5p is downregulated in NSCLCs and suppresses cell proliferation by direct inhibiting the expression of the oncogene CDK4[32]. MiR-486-5p is supported in its activities as a tumor suppressor in lung cancer by regulating insulin growth factor (IGF) signaling [33]. In studies on other cancers, miR-486-5p expression was decreased in gastric adenocarcinomas, esophageal squamous cell carcinomas, and colorectal carcinomas $[34,35]$. It is not unusual that one miRNA performs different functions in different cancer types. For instance, miR-486-5p is overexpressed in chronic myeloid leukemia and can enhance cell growth via regulation of AKT signaling and FOXO1 expression[36].

In conclusion, in the present study, SMAD2, as an important member of the TGF- $\beta$ signaling pathway, was proven to be targeted and downregulated by miR-486-5p in NSCLC cells. MiR-486-5p expression is downregulated in NSCLCs, whereas SMAD2 is upregulated. Overexpression of miR-486$5 p$ prevented TGF- $\beta$-induced EMT and cell migration and invasion in NSCLC cells. Our study provides new insights into the theoretical basis of NSCLC and therapeutic strategies that may be used to treat it.

\section{Acknowledgments}

We thank all patients who participated in this study. This work was supported by grants from the Science and Technology Plan Project of Suzhou (No. SYS201749), the Jiangsu Provincial Medical Youth Talent (No. QNRC2016746), the Societal and Developmental Project of Suzhou (No. SS201630), Natural science foundation of Jiangsu province (No. BK20180198), the Suzhou Key Laboratory for Respiratory Medicine (No. SZS201617), the Clinical Medical Center of Suzhou (No. Szzx201502), the Jiangsu Provincial Key Medical Discipline (No. ZDXKB2016007), and the Clinical Key Specialty Project of China.

\section{Competing Interests}

The authors have declared that no competing interest exists.

\section{References}

1. Li JJ, Li R, Wang W, Zhang B, Song X, Zhang C, et al. IDH2 is a novel diagnostic and prognostic serum biomarker for non-small-cell lung cancer. Molecular oncology. 2018; 12: 602-10.

2. Alidousty C, Baar T, Heydt C, Wagener-Ryczek S, Kron A, Wolf J, et al. Advance of theragnosis biomarkers in lung cancer: from clinical to molecular pathology and biology. Journal of thoracic disease. 2019; 11: S3-S8. 
3. Yu M, Chen Y, Li X, Yang R, Zhang L, Huangfu L, et al. YAP1 contributes to NSCLC invasion and migration by promoting Slug transcription via the transcription co-factor TEAD. Cell death \& disease. 2018; 9: 464.

4. Li Z, Zhang Y, Jin T, Men J, Lin Z, Qi P, et al. NQO1 protein expression predicts poor prognosis of non-small cell lung cancers. BMC cancer. 2015; 15 : 207.

5. Wrzesinski SH, Wan YY, Flavell RA. Transforming growth factor-beta and the immune response: implications for anticancer therapy. Clinical cancer research. 2007; 13: 5262-70.

6. Tang LY, Zhang YE. Non-degradative ubiquitination in Smad-dependent TGF-beta signaling. Cell \& bioscience. 2011; 1: 43

7. Du S, Bouquet S, Lo CH, Pellicciotta I, Bolourchi S, Parry R, et al. Attenuation of the DNA damage response by transforming growth factor-beta inhibitors enhances radiation sensitivity of non-small-cell lung cancer cells in vitro and in vivo. International journal of radiation oncology, biology, physics. 2015; 91: 91-9.

8. Murai F, Koinuma D, Shinozaki-Ushiku A, Fukayama M, Miyaozono K, Ehata S. EZH2 promotes progression of small cell lung cancer by suppressing the TGF-beta-Smad-ASCL1 pathway. Cell discovery. 2015; 1: 15026.

9. Wang L, Tong X, Zhou Z, Wang S, Lei Z, Zhang T, et al. Circular RNA hsa_circ_0008305 (circPTK2) inhibits TGF-beta-induced epithelial-mesenchymal transition and metastasis by controlling TIF1gamma in non-small cell lung cancer. Molecular cancer. 2018; 17: 140.

10. Lu Z, Li Y, Che Y, Huang J, Sun S, Mao S, et al. The TGFbeta-induced lncRNA TBILA promotes non-small cell lung cancer progression in vitro and in vivo via cis-regulating HGAL and activating S100A7/JAB1 signaling. Cancer letters. 2018; 432: 156-68

11. Mali AV, Joshi AA, Hegde MV, Kadam SS. Enterolactone modulates the ERK/NF-kappaB/Snail signaling pathway in triple-negative breast cancer cell line MDA-MB-231 to revert the TGF-beta-induced epithelial-mesenchymal transition. Cancer biology \& medicine. 2018; 15: 137-56.

12. Palma Cde S, Grassi ML, Thome CH, Ferreira GA, Albuquerque D, Pinto MT, et al. Proteomic Analysis of Epithelial to Mesenchymal Transition (EMT) Reveals Cross-talk between SNAIL and HDAC1 Proteins in Breast Cancer Cells. Molecular \& cellular proteomics : MCP. 2016; 15: 906-17.

13. Wang Y, Shang Y. Epigenetic control of epithelial-to-mesenchymal transition and cancer metastasis. Experimental cell research. 2013; 319: 160-9.

14. Lu J, Zhong Y, Chen J, Lin X, Lin Z, Wang N, et al. Radiation Enhances the Epithelial- Mesenchymal Transition of A549 Cells via miR3591-5p/USP33/PPM1A. Cellular physiology and biochemistry : international journal of experimental cellular physiology, biochemistry, and pharmacology. 2018; 50: 721-33.

15. Li H, Zhang Z, Chen L, Sun X, Zhao Y, Guo Q, et al. Cytoplasmic Asporin promotes cell migration by regulating TGF-beta/Smad2/3 pathway and indicates a poor prognosis in colorectal cancer. Cell death \& disease. 2019; 10: 109.

16. Rupaimoole R, Slack FJ. MicroRNA therapeutics: towards a new era for the management of cancer and other diseases. Nature reviews Drug discovery. 2017; 16: 203-22

17. Di Leva G, Garofalo M, Croce CM. MicroRNAs in cancer. Annual review of pathology. 2014; 9: 287-314.

18. Hayes J, Peruzzi PP, Lawler S. MicroRNAs in cancer: biomarkers, functions and therapy. Trends in molecular medicine. 2014; 20: 460-9.

19. Acunzo M, Romano G, Wernicke D, Croce CM. MicroRNA and cancer--a brief overview. Advances in biological regulation. 2015; 57: 1-9.

20. Xiao W, Zhong Y, Wu L, Yang D, Ye S, Zhang M. Prognostic value of microRNAs in lung cancer: A systematic review and meta-analysis. Molecular and clinical oncology. 2019; 10: 67-77.

21. Sun G, Ding X, Bi N, Wang Z, Wu L, Zhou W, et al. Molecular predictors of brain metastasis-related microRNAs in lung adenocarcinoma. PLoS genetics. 2019; 15: e1007888.

22. Yu S, Geng S, Hu Y. miR-486-5p inhibits cell proliferation and invasion through repressing GAB2 in non-small cell lung cancer. Oncology letters. 2018; 16: 3525-30.

23. Livak KJ, Schmittgen TD. Analysis of relative gene expression data using real-time quantitative PCR and the 2(-Delta Delta $\mathrm{C}(\mathrm{T})$ ) Method. Methods. 2001; 25: 402-8.

24. Zhu J, Zeng Y, Li W, Qin H, Lei Z, Shen D, et al. CD73/NT5E is a target of miR-30a-5p and plays an important role in the pathogenesis of non-small cell lung cancer. Molecular cancer. 2017; 16: 34.

25. Feng XH, Lin X, Derynck R. Smad2, Smad3 and Smad4 cooperate with Sp1 to induce p15(Ink4B) transcription in response to TGF-beta. The EMBO journal. 2000; 19: 5178-93.

26. Zhao BM, Hoffmann FM. Inhibition of transforming growth factor-beta1-induced signaling and epithelial-to-mesenchymal transition by the Smad-binding peptide aptamer Trx-SARA. Molecular biology of the cell. 2006; 17: 3819-31.

27. Zhang JX, Zhai JF, Yang XT, Wang J. MicroRNA-132 inhibits migration, invasion and epithelial-mesenchymal transition by regulating TGFbeta1/Smad2 in human non-small cell lung cancer. European review for medical and pharmacological sciences. 2016; 20: 3793-801.

28. Bao Y, Chen Z, Guo Y, Feng Y, Li Z, Han W, et al. Tumor suppressor microRNA-27a in colorectal carcinogenesis and progression by targeting SGPP1 and Smad2. PloS one. 2014; 9: e105991.
29. Wang C, Liu P, Wu H, Cui P, Li Y, Liu Y, et al. MicroRNA-323-3p inhibits cell invasion and metastasis in pancreatic ductal adenocarcinoma via direct suppression of SMAD2 and SMAD3. Oncotarget. 2016; 7: 14912-24.

30. Liu B, Sun J, Lei X, Zhu Z, Pei C, Qin L. MicroRNA-486-5p suppresses TGF-beta2-induced proliferation, invasion and epithelial-mesenchymal transition of lens epithelial cells by targeting Smad2. Journal of biosciences. 2017; 42: 575-84.

31. Zhang $X$, Zhang $T$, Yang $K$, Zhang M, Wang K. miR-486-5p suppresses prostate cancer metastasis by targeting Snail and regulating epithelial-mesenchymal transition. OncoTargets and therapy. 2016; 9: 6909-14.

32. Shao Y, Shen YQ, Li YL, Liang C, Zhang BJ, Lu SD, et al. Direct repression of the oncogene CDK4 by the tumor suppressor miR-486-5p in non-small cell lung cancer. Oncotarget. 2016; 7: 34011-21.

33. Peng Y, Dai $Y$, Hitchcock C, Yang X, Kassis ES, Liu L, et al. Insulin growth factor signaling is regulated by microRNA-486, an underexpressed microRNA in lung cancer. Proceedings of the National Academy of Sciences of the United States of America. 2013; 110: 15043-8.

34. Ren C, Chen H, Han C, Fu D, Zhou L, Jin G, et al. miR-486-5p expression pattern in esophageal squamous cell carcinoma, gastric cancer and its prognostic value. Oncotarget. 2016; 7: 15840-53

35. Kelley KA, Wieghard N, Chin Y, Potter A, Mori M, Wong MH, et al MiR-486-5p Downregulation Marks an Early Event in Colorectal Carcinogenesis. Diseases of the colon and rectum. 2018; 61: 1290-6.

36. Wang LS, Li L, Chu S, Shiang KD, Li M, Sun HY, et al. MicroRNA-486 regulates normal erythropoiesis and enhances growth and modulates drug response in CML progenitors. Blood. 2015; 125: 1302-13. 